\title{
The Mechanism of $\{113\}$ Defect Formation in Silicon: Clustering of Interstitial-Vacancy Pairs Studied by In Situ High-Resolution Electron Microscope Irradiation
}

\author{
Ludmila I. Fedina, ${ }^{1}$ Se Ahn Song, ${ }^{2, *}$ Andrey L. Chuvilin, ${ }^{3}$ Anton K. Gutakovskii, ${ }^{1}$ \\ and Alexander V. Latyshev ${ }^{1}$ \\ ${ }^{1}$ Institute of Semiconductor Physics, pr. Lavrentjeva 13, Novosibirsk, 630090, Russia \\ ${ }^{2}$ Quality Headquarters, Dongjin Semichen Co. Ltd., Hwaseong, 445-935, Korea \\ ${ }^{3}$ CIC nanoGUNE Consolider, Av. de Tolosa 76, 20018 San Sebastian, Spain
}

\begin{abstract}
We report the direct visualization of point defect clustering in $\{113\}$ planes of silicon crystal using a transmission electron microscope, which was supported by structural modeling and high-resolution electron microscope image simulations. In the initial stage an accumulation of nonbonded interstitial-vacancy (I-V) pairs stacked at a distance of $7.68 \AA$ along neighboring atomic chains located on the $\{113\}$ plane takes place. Further broadening of the $\{113\}$ defect across its plane is due to the formation of planar fourfold coordinated defects (FFCDs) perpendicular to chains accumulating I-V pairs. Closely packed FFCDs create a sequence of eightfold rings in the $\{113\}$ plane, providing sites for additional interstitials. As a result, the perfect interstitial chains are built on the $\{113\}$ plane to create an equilibrium structure. Self-ordering of point defects driven by their nonisotropic strain fields is assumed to be the main force for point defect clustering in the $\{113\}$ plane under the existence of an energy barrier for their recombination.
\end{abstract}

Key words: silicon $\{113\}$ defect, interstitial-vacancy clustering, self-ordering, fourfold coordinated defects, formation mechanism, in situ HR-TEM, defect modeling, HREM simulation

\section{INTRODUCTION}

One of the most important problems in the manufacture of submicron devices is the transient enhanced diffusion (TED) of implanted dopants in silicon during thermal annealing, which limits the size of the semiconductor devices (Napolitani et al., 2010). TED is believed to be mediated by interstitials (Is) stored in the shape of small clusters or extended $\{113\}$ defects. Hence, the study of interstitial clustering is considered the key for solving this problem. The equilibrium structure of the interstitial type $\{113\}$ defect has already been established (Takeda et al., 1994); however, its initial formation stage is still unclear. The most simple defect configuration containing an interstitial chain is obtained by chain insertion in the $\{111\}$ plane (Chou et al., 1995). However, this plane is not favorable for clustering of point defects; typically, only about $10 \%$ of the defects lie in the $\{111\}$ plane (Fedina et al., 1998). A few theoretical attempts were made to transform the interstitial chain in the $\{111\}$ plane to a $\{113\}$ defect through bond switching (Kim et al., 1997; Alippi \& Colombo, 2002). However, there is no experimental evidence substantiating such a transformation.

There are two main questions: What is the mechanism for interstitial chain formation on the $\{113\}$ plane, and why is the defect structure so complex? Ab initio calculations propose only compact configurations of small interstitial clusters (Kim et al., 2000; Richie et al., 2004). A new four-

() MICROSCOPY SOCIETY OF AMERICA 2013

${ }^{*}$ Corresponding author. E-mail: seahnsong@naver.com fold coordinated point defect (FFCD) corresponding to a closely bonded I-V pair was proposed as a building block for more extended defects (Goedecker et al., 2002). It is assumed that FFCD has not been detected experimentally yet. According to tight binding molecular dynamics (TBMD) and $a b$ initio calculations (Tang et al., 1997; Gargoni et al., 1998), the I-V pair is stable at room temperature because of an energy barrier for its recombination of $1.2-1.3 \mathrm{eV}$. In fact, we have already shown using in situ high-voltage electron microscope (HVEM; $1 \mathrm{MeV}$ ) and high-resolution electron microscope (HREM; $400 \mathrm{keV}$ ) irradiations that, at low temperatures $\left(20-350^{\circ} \mathrm{C}\right)$, self-interstitials and vacancies in Si tend to aggregate together mostly on $\{113\}$ planes (Fedina et al., 1997). Combined clustering of point defects provides the formation of a tiny amorphous phase, which is detected by diffraction pattern during in situ HVEM irradiation. After prolonged irradiation, the amorphous phase transforms into an equilibrium $\{113\}$ defect structure (Fedina et al., 1999).

Here we present the in situ study of $\{113\}$ defect evolution in a very thin Si sample during electron irradiation at room temperature.

\section{Materials and Methods}

The investigations were carried out using a medium-voltage high-resolution transmission electron microscope (TEM), JEOL-4000EX, operated at $400 \mathrm{keV}$, exceeding the threshold energy of $200 \mathrm{keV}$ for point defect creation in Si. The microscope is equipped with an objective lens pole piece 


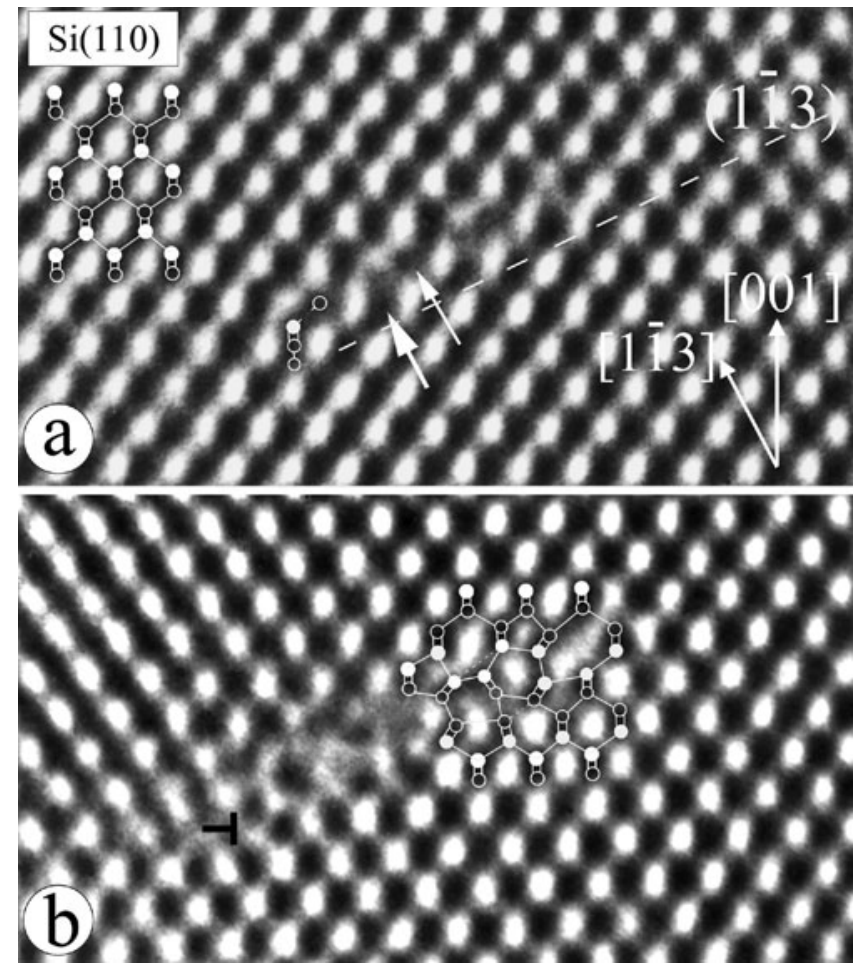

Figure 1. Evolution of the $\{113\}$ defect structure during in situ high-resolution electron microscope irradiation at room temperature: (a) $5 \mathrm{~min}$ and (b) $15 \mathrm{~min}$. The flux of electrons is about $10^{20} \mathrm{~cm}^{-2} \mathrm{~s}^{-1}$. For more details, see the text.

$\mathrm{UHP}-40 \mathrm{H}(\mathrm{Cs}=0.85 \mathrm{~mm}$ and point-to-point resolution of $1.6 \AA)$. The flux of electrons on a specimen during irradiation was estimated to be about $10^{20} \mathrm{~cm}^{-2} \mathrm{~s}^{-1}$ (see also the paper by Takeda \& Horiuchi, 1994). TEM specimens were prepared by chemical etching of (110) Fz-Si wafers implanted with boron ions at $30 \mathrm{keV}$ and at a dose of $10^{15} \mathrm{~cm}^{-2}$, followed by thermal annealing at $1,150^{\circ} \mathrm{C}$. High boron concentration was necessary to increase the rate of cluster nucleation in a very thin TEM sample (Aseev et al., 1994). Simulations of HREM images were performed with a multislice program MUSLI (Chuvilin \& Kaizer, 2005). Various defect structures were constructed within the $43 \times 42 \times$ $42 \AA$ supercell of $S i$ and were optimized by an $\mathrm{Mm}+$ force field. The differences between bond lengths in the defect configurations in our optimized models and the ones obtained by $a b$ initio calculations were within $0.1 \AA$ for the $\langle 110\rangle$-split interstitial and FFCD (Goedecker et al., 2002). For vacancies, the $\mathrm{Mm}+$ force field results in a very strong relaxation $(0.7 \AA)$, which is in fact no more than $0.2-0.3 \AA$ according to the first-principle calculations (Ogut \& Chelikovsky, 2001; Al-Mushadani \& Needs, 2003) and HREM data (Fedina et al., 1999, 2000a, 2000b). For this reason we introduced the vacancies in the models without optimization.

\section{Results AND Discussion}

Figures 1a and $1 \mathrm{~b}$ present experimental [110] HREM images of one and the same $\{113\}$ defect taken after 5 and 15 min of irradiation, respectively. In the first image, the defect is represented only by local variations in the length of the columns. Further, the defect becomes broader and obtains the features of an equilibrium structure outlined by a superimposed model. The left part of the final $\{113\}$ defect is disturbed by $60^{\circ}$ gliding dislocation; therefore, we will further consider only the right part as having a clear atomic structure. Images were obtained from a very thin crystal $(<100 \AA)$ close to the Scherzer focus, allowing straightforward interpretation: white spots correspond to channels in the Si lattice viewed in the [110] direction and the dark ones correspond to the projection of [110] atomic chains on the (110) plane.

The model of a perfect (110) Si crystal is superimposed with the HREM image in the left corner of Figure 1a. At the initial stage, the $\{113\}$ defect appears to form a sequence of paired neighboring columns located in the $\{113\}$ plane, one of which is elongated and the second one is shortened in the [001] direction (see the big and small arrows in Fig. 1). The column length deviation in both cases is about $40 \%$, in comparison with the columns of the perfect crystal. Such localized variations strongly suggest an accumulation of opposite-type point defects within the neighboring [110] atomic chains in the form of closely spaced IV pairs. According to the first-principle calculations, the most stable structure of the neutral self-interstitial atom is the split- $\langle 110\rangle$ configuration (Leung et al., 1999; Gharaibeh et al., 2001; Goedecker et al., 2002; Al-Mushadani \& Needs, 2003). The center of the split is shifted by about $0.76 \AA$ in the $\langle 100\rangle$ direction, and hence the split interstitial should extend the projection of that atomic chain along which the split direction is oriented to about $50 \%$. This agrees well with $40 \%$ column extension in the [001] direction detected by HREM. Therefore, we propose that experimental column extension is introduced by the split interstitial aligned along the [110] direction. In contrast, a vacancy should lead to shortening of atomic chain projection on the (110). A sketch of the atomic positions within the defective columns is shown in Figure 1a. However, single point defects are not considered to be detected by HREM. Hence, we should associate column length variations with multiple point defects stacked along the neighboring [110] atomic chains.

We have constructed several models with varying distances between the I-V pairs placed along [110] neighboring chains in second-neighbor positions. It was found that it is not possible to place split interstitials at a distance of $3.84 \AA$ and aligned along the chain direction because of the short space between split pairs providing their rotation from the exact [110] direction (model not shown). The model of defect chains with the distance of $7.68 \AA$ between I-V pairs viewed along the [110] and [110] directions is shown in Figures 2a and 2b. Split atoms are marked by red color (thin broken lines and balls present a schematic way of further I-V pair transformation). Arrows show positions of vacancies at neighboring atomic chains. Note that only five I-V pairs are placed along the [110] direction in the model in Figure 2b. The bond length between atoms forming split defects in our optimized model is $2.43 \AA$, which is similar to 


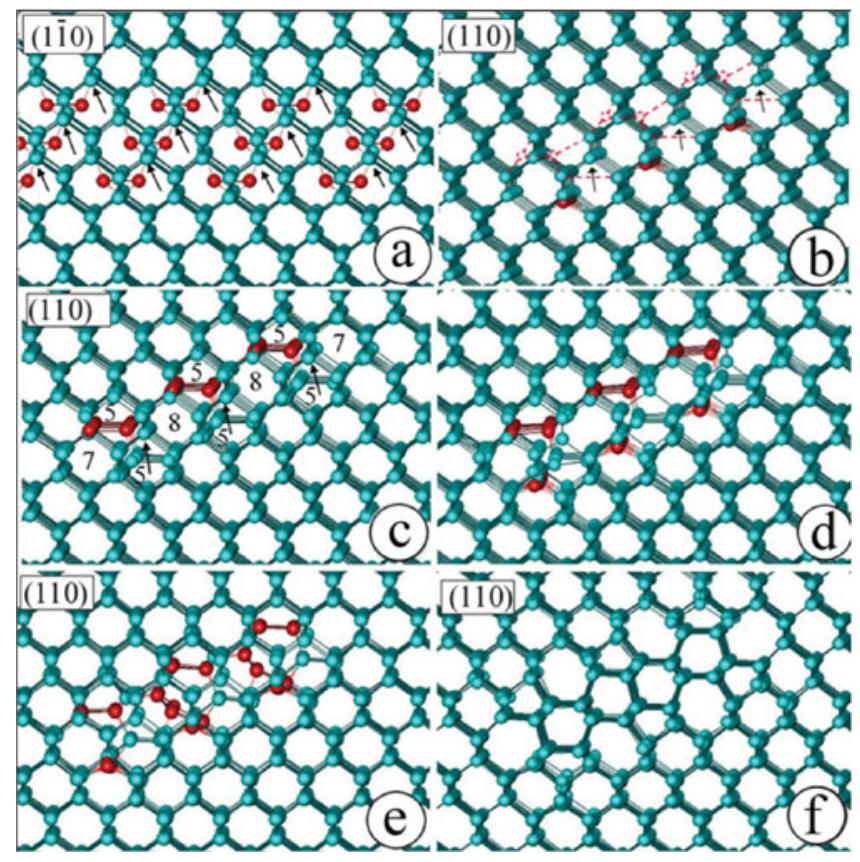

Figure 2. Models of evolution of the $\{113\}$ defect: (a,b) I-V pairs placed at $7.68 \AA$ in [110] chains and viewed along the ones (a) and end-on (b). c: Planar fourfold coordinated defects (FFCDs) stacked at a distance of $7.68 \AA$ along the [110] direction. d: Additional I-V pairs placed between stacked FFCDs. e: Same as (d), +10-atom interstitial chains inserted into each eightfold ring of FFCDs. f: Equilibrium structure of the $\{113\}$ defect. Red atoms are interstitials.

the value of $2.432 \AA$ found by $a b$ initio calculation (AlMushadani \& Needs, 2003). The bond lengths between split pairs and the nearest neighbor atoms vary from 2.24 to 2.6 $\AA$. The simulated HREM image of this model viewed in the [110] direction is presented in Figure 3a. In the experimental image in Figure 1a one can see that simulation gives a good reproduction of the extension and shortening of columns as explained previously.

If the distance between the $\mathrm{I}-\mathrm{V}$ pairs in the defect chains reached $15.36 \AA$, they were not visualized by HREM (a few percentages of column length deviations; image not shown). It may mean that to visualize $40 \%$ column length variations by HREM, the distance between I-V pairs should be at least $7.68 \AA$. At such a large distance an accumulation of rather nonbonded $\mathrm{I}-\mathrm{V}$ pairs takes place. There seems to be only one reason for this aggregation, which is based on self-ordering of point defects driven by their nonisotropic strain fields. Calculation of strain fields even for a single I-V pair is still an uphill task because it needs a large supercell as well as $a b$ initio methods. The silicon vacancy probably poses the greatest difficulty for $a b$ initio studies because the defect structure is dependent on convergence parameters and how the atomic relaxation is carried out (Al-Mushadani \& Needs, 2003). The experimental picture is more conclusive. Electron paramagnetic resonance studies have shown that the vacancy has $D 2 d$ symmetry, which is consistent with the Jahn-Teller distortion (Watkins, 1976). Low symmetry of point defect configurations suggests a

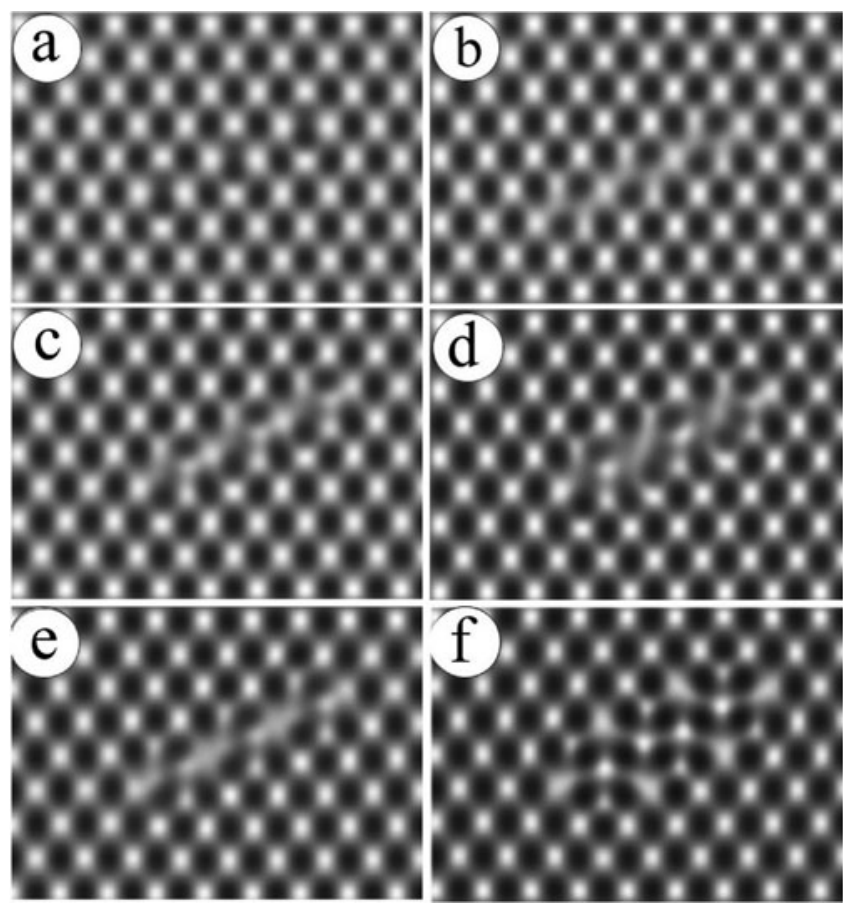

Figure 3. Simulated high-resolution electron microscope images of the evolution of the $\{113\}$ defect: (a-d) represent simulated images for the models Figures $2 \mathrm{~b}-2 \mathrm{e}$, respectively. e: Simulated image for the model Figure $2 \mathrm{c}$ with an increased amount (8) of stacked fourfold coordinated defects along the [110] direction. f: Simulated image for the model Figure 2f. The crystal thickness and defocus value are 43 and $-360 \AA$, respectively.

nonisotropic strain field distribution around them and the possibility of strain field compensations by close location of point defects at neighboring chains. As a result, the $\mathrm{I}-\mathrm{V}$ pair becomes stable before FFCD formation and can be visualized by HREM.

According to TBMD simulation (Tang et al., 1997), the reason for FFCD formation instead of I-V annihilation is the local distortion around the $\langle 110\rangle$-split atom induced by the close approach to the vacancy. Spontaneous recombination occurs only for I-V pairs separated by first and second nearest-neighbor distances along the $\langle 110\rangle$ split direction. In all other directions corresponding to the positions of the split interstitial and vacancy at neighboring chains, FFCDs should form upon their close contact. These TBMD results are in excellent agreement with the HREM observation of $\mathrm{I}-\mathrm{V}$ pair accumulation in neighboring chains, resulting in further FFCD formation. Figure $1 \mathrm{~b}$ shows that after several minutes of irradiation a clear broadening of the $\{113\}$ defect across its plane is observed. The broadening takes place on the (110) plane, which is perpendicular to the direction of chains accumulating I-V pairs. The positions of two five and sevenfold rings at the edge of the $\{113\}$ defect exactly correspond to the model of single FFCD proposed by TBMD simulation (Tang et al., 1997). Closely spaced I-V pairs, however, produce a sequence of eightfold rings on the $\{113\}$ plane instead of sevenfold ones corresponding to a single FFCD. 
On the basis of the HREM data and an initial model of $\mathrm{I}-\mathrm{V}$ pairs shown in Figures $2 \mathrm{a}$ and $2 \mathrm{~b}$, we constructed the model of FFCDs on the $\{113\}$ plane stacked by $7.68 \AA$ along the [110] direction (Fig. 2c). A schematic of planar FFCD formation is shown by thin red broken lines in Figure 2b. To create FFCDs on the (110) plane, each interstitial atom should change its position, followed by changing the split direction from [110] to [11̄0]. According to TBMD data (Tang et al., 1997), this is necessary to place the IV pair along the same atomic chain laying on the (110) plane and to make bond rearrangements leading to FFCD. Figure $2 \mathrm{c}$ presents such an optimized model of FFCDs on $\{113\}$. Bond lengths characterizing FFCDs in our model vary from 2.24 to $2.5 \AA$ and are very similar to the values $2.25-2.47 \AA$ found in the studies by Goedecker et al. (2002) and AlMushadani and Needs (2003). From the image simulation we have found that the distanced FFCDs are not visualized by HREM, and only small shortening of columns located on the $\{113\}$ plane is observed (see Fig. 3b). Nevertheless, formation of the distanced planar FFCDs allows additional $\mathrm{I}-\mathrm{V}$ pairs to be inserted along the initial atomic chains (see Fig. 2d). In this case the best fit of column shortening to the initial experimental image of the $\{113\}$ defect is obtained by HREM image simulation (Fig. 3c). At the same time, large eightfold rings created by closely spaced FFCDs on $\{113\}$ may provide the sites for an additional insertion of interstitials. Figure 2e presents the model of stacked FFCDs with additional I-V pairs placed between them and with tenatom interstitial chains inserted into eightfold rings. The simulated HREM image in this case becomes similar to the experimental one at the final stage, although not identical (Fig. 3d). Further increase in the number of stacked FFCDs leads to an appearance of a large bright spot in the simulated HREM image corresponding to eightfold rings in the $\{113\}$ plane (Fig. 3e). The model in Figure $2 \mathrm{f}$ corresponds to the stacked FFCDs at each (110) plane with complete interstitial chains inserted into eightfold rings. The HREM image simulated by this model and shown in Figure $3 \mathrm{f}$ agrees well with the one observed by Takeda for the small $\{113\}$ defect (in his PEIIEP notation) introduced by $2-\mathrm{MeV}$ irradiation at $450^{\circ} \mathrm{C}$ (Takeda et al., 1994). Such an image of the $\{113\}$ defect is not realized during $15 \mathrm{~min}$ of HREM irradiation at room temperature.

Several additional models have been tested by HREM image simulations and it was revealed that only some combinations of stacked FFCDs with $\mathrm{I}-\mathrm{V}$ pairs between them and with interstitials inserted into eightfold rings provide good agreement between experimental and calculated images of $\{113\}$ defect in our case. Figure 4 presents the initial and final experimental HREM images in comparison with simulated ones and optimized models used for simulation. In the model at an initial stage the number of I-V pairs along the [110] atomic chains dominates; three distanced layers of FFCDs on $\{113\}$ with six-atom chains inserted into each eightfold ring are not seen in the simulated image (Fig. $4 \mathrm{~b}$ ). At the final stage, the number of FFCD layers is increased up to eight and they are clearly seen in the image together with

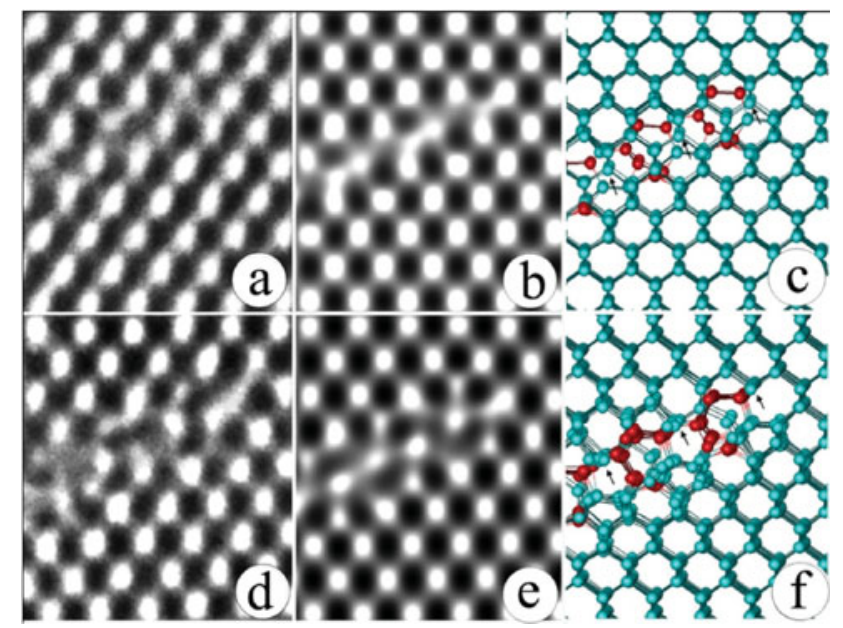

Figure 4. High-resolution electron microscope (HREM) images and models of the $\{113\}$ defect in the initial and final stages. a,b: Experimental and simulated HREM images at the initial stage. c: A model consisting of five stacked I-V pairs and three fourfold coordinated defects (FFCDs) per two $\langle 110\rangle$ neighboring atomic chains on the $\{113\}$ plane and six atoms inserted into eight rings. d,e: Experimental and simulated HREM images at the final stage. $\mathrm{f}$ : A model consisting of eight stacked FFCDs and three I-V pairs with interstitial chains consisting of ten (left) and 16 atoms (right).

interstitial chains inserted into FFCDs (Fig. 4e). Taking into account both an absence of vacancy relaxation in our models and a low relaxation rate of the defect structure at room temperature one can see that good correspondence is reached between experimental and simulated images.

\section{CONCLUSION}

We conclude that the mechanism of $\{113\}$ defect formation in $\mathrm{Si}$ is based on combined aggregation of self-interstitials and vacancies. At an initial stage an accumulation of mostly nonbonded I-V pairs stacked at a distance of $7.68 \AA$ along neighboring atomic chains located on the $\{113\}$ plane takes place. The key step of $\{113\}$ defect formation providing its complex structure is the formation of planar FFCD perpendicular to the direction of chains accumulating I-V pairs. This allows additional I-V pairs to be inserted along initial atomic chains in order to form the stacked FFCDs at each (110) plane. Closely spaced FFCDs on the $\{113\}$ plane create a sequence of eightfold rings that become the sites for an additional insertion of supersaturated interstitials. As a result, perfect interstitial chains are built on the $\{113\}$ plane to create an equilibrium structure.

\section{ReFERENCES}

Alippi, P. \& Cоцомво, L. (2002). Lattice-strain field induced by $\{311\}$ self-interstitial defects in silicon. Phys Rev B 62, 1815-1820. Al-Mushadani, O.K. \& Needs, R.J. (2003). Free-energy calculations of intrinsic point defects in silicon. Phys Rev $B$ 68, 235205-235213.

Aseev, A., Fedina, L., Hoenl, D. \& Barsch, H. (1994). Clusters of Interstitial Atoms in Silicon and Germanium. Berlin, Germany: Academy Verlag. 
Chou, C.T., Cockayne, D.J.H., Zou, J., KringhoJ, P. \& Jagadish, C. (1995). $\{111\}$ defects in 1-MeV-silicon-ion-implanted silicon. Phys Rev B 52, 17223-17230.

Chuvilin, A. \& Kaizer, U. (2005). On the peculiarities of CBED pattern formation revealed by multislice simulation. Ultramicroscopy 104, 73-82.

Fedina, L., Gutakovskit, A. \& Aseev, A. (2000a). In situ HREM irradiation study of an intrinsic point defects clustering in FZ-Si. Cryst Res Technol 35, 775-786.

Fedina, L., Gutakovskit, A., Aseev, A., Van Landuyt, J. \& Vanhellemont, J. (1997). The intrinsic point defect clustering in Si: A study by HVEM and HREM in situ electron irradiation. In In Situ Microscopy in Material Research, Gai, P. (Ed.), p. 63. Dordrecht: Kluwer.

Fedina, L., Gutakovskit, G., Aseev, A., Van Landuyt, J. \& Vanhellemont, J. (1998). On the mechanism of $\{111\}$-defect formation in silicon studied by in situ electron irradiation in a high resolution electron microscope. Phil Mag A 77, 423-435.

Fedina, L., Gutakovskif, A., Aseev, A., Van Landuyt, J. \& VanHELlemont, J. (1999). Extended defects formation in Si crystals by clustering of intrinsic point defects studied by in-situ electron irradiation in an HREM. Phys Stat Sol (a) 171, 147-158.

Fedina, L., Lebedev, O.I., Van Tendeloo, G., Van Landuyt, J., Mironov, O.A. \& PARker, E.H.C (2000b). In situ HREM irradiation study of point-defect clustering in MBE-grown strained $\mathrm{Si}_{1-\mathrm{x}} \mathrm{Ge}_{\mathrm{x}} /(001)$ Si structures. Phys Rev B 61, 10336-10345.

Gargoni, F., Gatti, C.L. \& Colombo, L. (1998). Intrinsic point defects in crystalline silicon: Tight-binding molecular dynamics studies of self-diffusion, interstitial-vacancy recombination, and formation volumes. Phys Rev B 57, 170-177.

Gharaibeh, M., Estreicher, S.K., Fedders, P.A. \& Ordejón, P. (2001). Self-interstitial-hydrogen complexes in silicon. Phys Rev B 64, 235211-1-235211-7.

Goedecker, S., Deutsch, T. \& Billard, L. (2002). A fourfold coordinated point defect in silicon. Phys Rev Lett 88, 235501-235504.
Kim, J., Kirchhoff, F., Wilkins, J.W. \& Khan, F.S. (2000). Stability of Si-interstitial defects: From point to extended defects. Phys Rev Lett 84, 503-506.

Kim, J., Wilkins, J.W., Khan, F.S. \& Canning, A. (1997). Extended Si $\{311\}$ defects. Phys Rev B 55, 16186-16197.

Leung, W.K., Needs, R.J., Rajagopal, G., Itoh, S. \& Ihara, S. (1999). Calculations of silicon self-interstitial defects. Phys Rev Lett 83, 2351-2354.

Napolitani, E., Bisognin, G., Bruno, E., Mastromatteo, M., Scapellato, G.G., Boninelli, S., De Salvador, D., MiraBella, S., Spinella, C., Carnera, A. \& Priolo, F. (2010). Transient enhanced diffusion of B mediated by self-interstitials in preamorphized Ge. Appl Phys Lett 96, 201906-201909.

Ogut, S. \& Chelikovsky, J.R. (2001). Ab initio investigation of point defects in bulk $\mathrm{Si}$ and Ge using a cluster method. Phys Rev B 64, 245206-245217.

Richie, D.A, Kim, J., Barr, S.A., Hazzard, K.R.A., Hennig, R. \& WiLKINs, J.W. (2004). Complexity of small silicon self-interstitial defects. Phys Rev Lett 92, 45501-45504.

TAKeda, S. \& Horiuchi, S. (1994). Electron diffraction channeling effect on defect formation in Si with 110 zone axis incidence. Ultramicroscopy 56, 144-162.

Takeda, S., Kohyama, M. \& Ibe, K. (1994). Interstitial defects on $\{113\}$ in Si and Ge: Line defect configuration incorporated with a self-interstitial atom chain. Phil Mag A 70, 287-312.

Tang, M., Colombo, L., Zhu, J. \& Diaz de la Rubia, T. (1997). Intrinsic point defects in crystalline silicon: Tight-binding molecular dynamics studies of self-diffusion, interstitialvacancy recombination, and formation volumes. Phys Rev B 55, 14279-14289.

WATKINS, G.D. (1976). EPR studies of lattice defects in semiconductors. In Defects and Their Structure in Non Metallic Solids. Henderson, B. \& Hughes, A.E. (Eds.), p. 203. New York: Plenum Press. 